\title{
The Absorption of Cetylpyridinium Chloride into PolyNIPAM-based Microgel Particles, in Dispersion and as Surface-Deposited Monolayers
}

\author{
Verawan Nerapusri ${ }^{1}$, Joseph L. Keddie ${ }^{2}$, Brian Vincent ${ }^{1, *}$ and Ibraheem A. Bushnak ${ }^{2}$ \\ ${ }^{1}$ School of Chemistry, University of Bristol, Bristol, BS8 1TS, UK \\ ${ }^{2}$ Department of Physics, University of Surrey, Guildford, GU2 7XH, UK
}

author to whom correspondence should be addressed: brian.vincent@bris.ac.uk

keywords: microgels, monolayers, ellipsometry, poly( $\mathrm{N}$-isopropylacrylamide), cetylpyridinium chloride, controlled release.
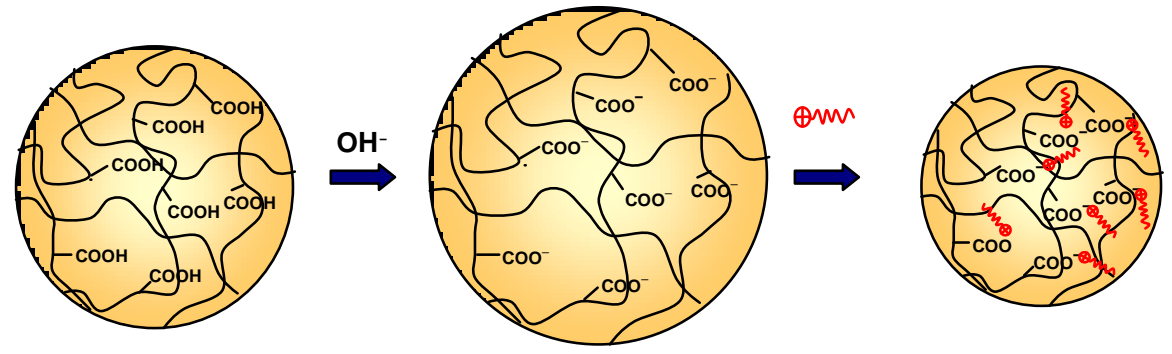

table of contents picture 


\begin{abstract}
The addition of cetylpyridinium chloride [CPC] to aqueous dispersions of poly $(\mathrm{N}-$ isopropylacrylamide) [poly(NIPAM)] and of poly(N-isopropylacrylamide-co-acrylic acid) [poly(NIPAM-co-AAc)] microgel particles leads to absorption of the CPC into the particles and to corresponding changes in their hydrodynamic diameter. With the latter set of particles there is a strong $\mathrm{pH}$-dependence. The dependence of both the hydrodynamic diameter and the electrophoretic mobility of the microgel particles on the added CPC concentration show a strong correlation with CPC uptake, as obtained from direct CPC absorption measurements. Various mechanisms for CPC absorption into the microgel particles are postulated, including electrostatic, polar and hydrophobic interactions.

A comparison has also been made between the effect of added CPC on the hydrodynamic diameter of the free microgel particles in dispersion determined using dynamic light scattering, and the thickness of adsorbed monolayers of the same microgel particles deposited on cationically-modified, oxidised silicon surfaces, as determined from ellipsometry measurements. The trends observed in both cases are broadly similar.

This work opens the way for the development of microgel layers for controlled uptake and release applications.
\end{abstract}




\section{Introduction}

Dispersions of microgel particles have been widely studied [1-3], in particular, those based on poly (N-isopropylacrylamide) [poly(NIPAM)]. Such microgel particles swell or deswell with changes in their environmental conditions (e.g. temperature or solvent type). If ionisable comonomer groups are introduced (e.g. acrylic acid) [AAc] then microgel particles also respond to changes in $\mathrm{pH}$ or electrolyte concentration. For this reason microgel particles have been proposed for uses in many applications, such as controlled uptake and release [1-3]. Indeed, this potential has been investigated in a number of earlier studies by the Bristol group, for various kinds of model "active" species, ranging from metal ions [4,5] and surfactants [6,7] to polymers [8,9] and nanoparticles [10]. In all cases it was demonstrated that the active species are absorbed into the interior of the microgel particles. For larger species such as polymers [8] and nanoparticles [10], the extent of their penetration is limited by the cross-link density of the polymer network comprising the particles. The size of the species concerned, compared to the average "mesh size" within the microgel particles, clearly then comes into consideration.

In this paper we report the absorption of the cationic surfactant cetylpyridinium chloride [CPC] into two sets of microgel particles: (i) poly(NIPAM), which have an anionic surface charge, associated with the initiator (potassium persulfate) residues; and (ii) poly(NIPAM-co-AAc, which also develop a bulk anionic charge, at $\mathrm{pH}$ values above $\sim 4$ due to ionisation of the $-\mathrm{COOH}$ groups. This work extends our earlier studies of the absorption of non-ionic surfactants into similar particles [6], and also of the absorption of the photo-degradable anionic surfactant 4-hexylphenylazosulfonate into (cationic) poly(2-vinylpyridine) microgel particles [7]. Tam et al. [11], have previously shown that cationic surfactants do adsorb into poly(NIPAM) microgel particles carrying cationic surface charge groups, and, similarly, anionic surfactants do adsorb into poly(NIPAM) microgel particles carrying anionic charge groups, although non-ionic surfactants adsorbed into neither type of microgel particle. Other studies [12-14] have been made of the uptake of the anionic surfactant, sodium dodecyl sulfate, into NIPAM-based microgel particles. Densmore et al. [15] have studied the swelling / deswelling behaviour of poly(hydroxyethylmethacrylate) microgel particles in the presence of CPC. 
Clearly, when the surfactant and microgel particles have the same charge sign, as in the work of Tam et al. [11], then the primary driving force for the absorption of surfactant molecules is the hydrophobic interaction between the hydrocarbon tails of the surfactant and hydrophobic moeities within the microgel particles. When the two charge signs are opposite, however, as in the present studies, then the electrostatic attraction between the surfactant head-groups and the oppositely-charged moeities within the microgel particles may be expected to play a more significant role.

In this current work the swelling / deswelling behaviour of the two sets of (anionic) NIPAM-based microgel particles referred to earlier, in the presence of CPC, has been monitored using dynamic light scattering. Complementary studies of the electrophoretic mobility of the microgel particles have been made, and also the absorption isotherms of CPC into the microgel particles have been established using the solution depletion analysis method, coupled to UV-visible spectroscopic determination of the CPC concentration in bulk solution.

In certain applications, it would be better to have the microgel particles deposited onto a substrate, as a monolayer on the surface. Examples might be in the release of bactericides into food processing lines, or even release from teeth and gum surfaces in the mouth. Microgel monolayers might even be deposited on larger, hard support particles for use in packed-columns for selective uptake and separation purposes. In a previous paper [16], as a first step to investigating these possible applications, we described a simple "dipping" procedure for the formation of close-packed monolayers of poly(NIPAM) and poly(NIPAM-co-AAc) microgel particles on cationically-modified silicon wafers. The silicon wafers had been rendered cationic by first oxidising their surface to silica and then adsorbing a layer of poly(ethyleneimine). The thickness of the deposited microgel monolayers was determined using spectroscopic ellipsometry. In that earlier paper [16] changes in the thickness were followed as a function of temperature, $\mathrm{pH}$ and added $\mathrm{NaCl}$ concentration In the current paper we extend these studies to follow the thickness changes with added CPC concentration. The thickness of the monolayer is compared with the hydrodynamic diameter of the free microgel particles, dispersed in aqueous solution, under similar environmental conditions (in particular $\mathrm{pH}$ ). 


\section{Experimental Section}

\section{Materials}

1-hexadecylpyridinium (i.e. cetylpyridinium) chloride [CPC] monohydrate (Acros, 96\%) was used as supplied. Water was Purite "Milli-Q" grade (with a resistivity of $18.2 \mathrm{M} \Omega \mathrm{cm}$ ). Poly(ethyleneimine) [PEI] was obtained from $\mathrm{BDH}$, as a $50 \mathrm{wt} \%$ aqueous solution. Potassium chloride $[\mathrm{KCl}]$ was also obtained from $\mathrm{BDH}$.

\section{The Microgel Dispersions}

The poly(NIPAM) and poly(NIPAM-co-AAc) microgel particles used in this work were identical to those described in an earlier paper by us [16]. The reader is referred to that paper for details of their preparation and clean-up. They were both prepared by surfactant-free emulsion polymerisation, essentially following the original procedure described by Pelton and Chibanate [17].

\section{Hydrodynamic Diameters and Electrophoretic Mobilities of the Microgel Particles}

The diffusion coefficients and electrophoretic mobilities of the microgel particles were determined by dynamic light scattering [DLS] and phase analysis light scattering [PALS], respectively, using a Brookhaven Instruments Zeta Plus apparatus, fitted with a $15 \mathrm{~mW}$ laser $(\lambda=678 \mathrm{~nm})$. Samples were prepared at a particle concentration of $0.025 \mathrm{wt} \%$, and were checked to ensure no multiple scattering was present. The StokesEinstein equation was then used to calculate the hydrodynamic diameter of the particles from the diffusion coefficient. The solids content of the microgel preparations were determined gravimetrically. The $\mathrm{pH}$ was adjusted by the addition of small quantities of $\mathrm{HCl}$ or $\mathrm{NaOH}$.

\section{Absorption and Release of CPC by the Microgel Particles}

The absorbed amount of CPC by the microgel particles, for a given initial CPC concentration, was determined using the classical solution concentration-depletion 
method. Microgel dispersions and CPC solutions, both of known initial concentration, were mixed and left to equilibrate for $24 \mathrm{hr}$. The dispersion was then centrifuged at 10,000 rpm, for $15 \mathrm{~min}$, using a Sorvall SA600 centrifuge. The equilibrium CPC concentration in the supernatant was determined at $260 \mathrm{~nm}$ using a Hewlett-Packard Agilent 8453E UV-Visible spectrometer.

A similar procedure was used to determine the released amount of CPC into the bulk solution after reducing the $\mathrm{pH}$ of the dispersion from $\mathrm{pH} 8$ to 3 .

\section{Deposition of Microgel Monolayers on Macroscopic Surfaces}

The basic details are described in our previous paper [16]. The substrates were silicon wafers, which, prior to use, were cleaned with $\mathrm{HCl} / \mathrm{H}_{2} \mathrm{O}_{2}$ solution $(70: 30 \mathrm{vol} \%)$ at $80{ }^{\circ} \mathrm{C}$ for $1 \mathrm{~h}$, followed by rinsing with purified water 10 times. This procedure also leads to oxidization of the silicon surface, to produce a thin silica layer at the surface, whose net surface charge is then $\mathrm{pH}$-dependent. In order to render the surface charge net positive at neutral $\mathrm{pH}$, the silicon wafer was dipped into a $10 \mathrm{wt} \%$ PEI solution (for $5 \mathrm{~min}$ ) and again thoroughly rinsed with water. Streaming potential data for the bare and PEIcovered surfaces, as a function of $\mathrm{pH}$, were presented previously [16].

The negatively-charged microgel particles were then deposited onto this cationically-modified silicon wafer by a simple dipping procedure. The wafer was dipped into the required microgel dispersion, at $50{ }^{\circ} \mathrm{C}$, for $5 \mathrm{~min}$ to allow maximum coating of the substrate surface to occur. The wafer was then transferred to water, at room temperature, for a further $15 \mathrm{~min}$. The microgel-coated wafer was allowed to dry in a clean air environment. The characterisation (SEM and ellipsometric thickness measurements) of the dried monolayers of microgel particles has been described in detail previously [16].

\section{Spectroscopic Ellipsometry Analysis.}

Ellipsometry is a technique that has been widely used to determine the thickness $(\delta)$ and refractive index (n) of an adsorbed or deposited thin film on an optically-flat surface, for many types of soft matter [18], including polymers [19-22]. The principles of 
the technique may be found elsewhere [23, 24]. In brief, ellipsometry measures the change in the polarization state of light caused by the reflection from a single interface, a series of parallel interfaces or a gradient interface. The technique measures the two ellipsometry parameters, $\Delta$ and $\Psi$, as a function of wavelength and / or angle-ofincidence. These parameters are related to the ratio of the Fresnel reflection coefficients, $\mathrm{R}_{\mathrm{p}}$ and $\mathrm{R}_{\mathrm{s}}$, for $\mathrm{p}$ and s-polarized light, respectively, through

$$
\tan \Psi \cdot \mathrm{e}^{\mathrm{i} \Delta}=\mathrm{R}_{\mathrm{p}} / \mathrm{R}_{\mathrm{s}}
$$

Ellipsometry measurements were performed using a variable-angle spectroscopic instrument (J.A. Woollam Co. Inc., USA) with a rotating analyser configuration. The chosen wavelengths ranged from 400 to $800 \mathrm{~nm}$, where both poly(NIPAM) and CPC are optically transparent. The ellipsometry spectra of the silicon oxide layer, polyelectrolyte (PEI) layer, and dry microgel layer were first obtained, via successive measurements, in air. The thickness and refractive index values of each of the layers were thereby independently obtained, as already reported [16]. These values were then used in subsequent analysis. The silicon wafer, with the adsorbed microgel layer, was placed in a liquid cell, having permanent glass windows, less than $150 \mu \mathrm{m}$ in thickness [21]. The liquid cell was placed on a thermostatting unit that allowed solution temperature control to within $\pm 0.1{ }^{\circ} \mathrm{C}$. Measurements were performed with the incident light beam set at $72^{\circ}$ with respect to the sample normal, such that the incident and reflected light beams were normal to the glass windows.

The $\delta$ and $n$ values for the microgel layers were derived from the $(\Psi, \Delta)$ spectra using a model-dependent fitting procedure [24]. The data were fitted with software in an iterative procedure using a Marquardt algorithm to reduce the mean-square error between the experimental and simulated data [25]. In the model, each layer (including the microgel layer) was treated as a planar slab with a uniform refractive index. In this fitting procedure, only $\delta, \mathrm{n}$ and thickness non-uniformity (i.e. a percentage variation in thickness across the footprint of the beam) were treated as fitting parameters. Although a slab is a simplified description for a microgel layer, a more complex model with an arbitrary gradient in refractive index with depth into the layer would introduce too many unknown parameters and thus cannot be justified. An alternative approach would be to 
assume a functional form for the refractive index profile. However, that requires prior knowledge of the structure. In dilute dispersions, small-angle neutron scattering data were consistent with a uniform core and a gradient shell structure [26], but an analogous multi-layer and/or gradient layer model for the microgel monolayer does not significantly improve the quality of the fitting, making a more complex model unsupportable. Moreover, the primary objective of the measurements is to determine the conditions under which microgel layers swell and de-swell, and the slab model is sufficient to achieve this objective. In this analysis, $\delta$ should be considered to be an apparent thickness for the layer.

Previous electron microscopy of the dry microgel monolayers [16] has revealed dense-packing of the microgel particles within the monolayer. However, a few packing defects and vacancies were observed. The footprint of the ellipsometer's light beam is several $\mathrm{mm}^{2}$. Any defects or vacancies smaller than the wavelength of light contribute to a reduced average $n$ for the monolayer. Any larger non-uniformities are considered through the thickness non-uniformity assumed in the model. Herein, only the average values for $\delta$ will be reported.

\section{Results and Discussion}

\section{Electrophoretic Mobility of Dispersed Microgel Particles with added CPC}

The electrophoretic mobility values of both the poly(NIPAM) and the poly(NIPAM-co-AAc) microgel particles, as a function of added CPC concentration, at $\mathrm{pH} 3$ or 8 , at $20^{\circ} \mathrm{C}$ and in $10^{-3} \mathrm{M} \mathrm{KCl}$ background solution, are shown in Figure 1. It may be seen that the electrophoretic mobility, in all the cases studied, is initially negative. Then, as the CPC concentration is increased, the electrophoretic mobility decreases initially, passes through zero, and then becomes increasingly positive. For the poly(NIPAM-co-AAc) microgel particles at $\mathrm{pH} 8$ no measurements were possible for added $\mathrm{CPC}$ concentrations in the range 0.4 to $0.8 \mathrm{mM}$ because the particles were aggregated Nevertheless, for this system the reversal of charge at added CPC concentrations greater than $0.8 \mathrm{mM}$ may be clearly observed. It should be noted that the 
critical micelle concentration $(\mathrm{cmc})$ of free CPC molecules in aqueous solution is $0.6 \mathrm{mM}$ [27]. It would seem, therefore, that this charge reversal occurs in the region of the cmc.

It is interesting that the three plots in Figure 1 are almost coincident. There is really no rigorous theory available in the literature for analysing the electrophoretic mobility of microgel particles with absorbed surfactant. However, intuitively, it seems likely that the net surface charge contribution (i.e. the net charge in the periphery region of the particles) largely determines the mobility value. The most pronounced change in mobility (from negative to positive) occurs at low CPC concentrations. It is reasonable to assume that the initially adsorbed CPC molecules will be located in this periphery region, mostly balancing the strong, negatively-charged (sulfate) groups arising from the initiator. Since the density of these sulfate groups is likely to be similar for the three systems shown in Figure 2, then the resulting similarity of the mobility results can rationalised in this way.

\section{Hydrodynamic Diameter of Dispersed Microgel Particles with added CPC}

The equilibrium hydrodynamic diameters of both the poly(NIPAM) and the poly(NIPAM-co-AAc) microgel particles, as a function of added CPC concentration, at $\mathrm{pH} 3$ or 8 and at $20^{\circ} \mathrm{C}$, are shown in Figure 2. It may be seen that for the poly(NIPAM) and the poly(NIPAM-co-AAc) microgel particles at $\mathrm{pH} \mathrm{3,} \mathrm{the} \mathrm{hydrodynamic} \mathrm{diameter}$ increases with increasing CPC concentration. The most pronounced increase in swelling occurs just beyond the cmc of free CPC molecules in aqueous solution $(0.6 \mathrm{mM}$, see section 1). Since the microgel particles are already swollen in the absence of the CPC molecules, these observations must imply that CPC molecules are absorbed into the microgel particles (possibly as micellar-type aggregates), and it is this effect which is causing their further swelling. The main cause of the swelling beyond the cmc is clearly the build-up of net positive charge within the microgel particles, as can be seen from Figure 1. However, there may be a secondary contributing factor. We showed in an earlier paper from this laboratory [6] that the absorption of non-ionic surfactants into similar NIPAM-based microgel particles also leads to swelling (at least up to the point where the microgel particles become saturated with the surfactant). Microgel particles 
may be considered to contain "pores" or "cages", formed by random-coil, oligomeric chains, linked together at the cross-links. A micellar-like aggregate, of similar or slightly larger dimensions to these cages, will tend to expand the cage volume through a kind of "steric" effect; this may also contribute to the net swelling observed in Figure 2. There is evidence in the literature, from small-angle neutron scattering studies [28], for the existence of such micellar-like aggregates within microgel particles, although they would appear to be smaller in size than the equivalent, "normal" micelles in aqueous solution.

For the poly(NIPAM) microgel particles, and also the poly(NIPAM-co-AAC) microgel particles at $\mathrm{pH} 3$, the maximum increases in size, attained at high CPC concentrations $(\sim 2.5 \mathrm{mM})$, are $35 \%$ and $75 \%$, respectively. Since, under the specified conditions, the interiors of both of these sets of microgel particles carry no bulk charge groups (only surface charge groups from the initiator residues) the primary driving force for the absorption of CPC is most likely hydrophobic bonding between the tails of the surfactant molecules and the isopropyl moieties within the microgel particle interiors. However, given the fact that, in relative terms, the poly(NIPAM-co-AAC) microgel particles (at $\mathrm{pH} 3$ ) swell more than the poly(NIPAM) microgel particles, it would appear that in the former system an additional driving force for CPC absorption may be present; this could be some form of polar interaction between the cationic pyridinium head groups of the surfactant molecules and the $-\mathrm{COOH}$ moieties within the microgel particles.

For the poly(NIPAM-co-AAC) microgel particles, at $\mathrm{pH} \mathrm{8,} \mathrm{where} \mathrm{the} \mathrm{AAc}$ moieties are now in the ionised $\left(\mathrm{COO}^{-}\right)$form, a rather different result is observed in Figure 2. With increasing CPC concentration the particle size first decreases, to some minimum value, at an added CPC concentration in the region of the $\mathrm{cmc}$ value $(0.6 \mathrm{mM})$. The minimum size attained could not actually be determined because, as explained in section 1, the microgel particles aggregated over the CPC concentration range from 0.4 to $0.8 \mathrm{mM}$. The primary driving force for absorption of CPC molecules, at lower CPC concentrations, is the electrostatic attraction between the cationic pyridinium head groups of the surfactant molecules and the anionic $\mathrm{COO}^{-}$moieties within the microgel particles. This neutralisation of the charge groups within the microgel particles leads to their observed deswelling. From Figure 2 it may be seen that, at added concentrations of CPC greater than $0.8 \mathrm{mM}$, the poly(NIPAM-co-AAC) microgel particles at $\mathrm{pH} 8$ begin to 
reswell. This is because further absorption of CPC now causes a build-up of net cationic bulk charge within the microgel particles, as may be seen in Figure 1.

\section{Absorption Isotherms}

Figure 3 shows the equilibrium absorption isotherms for CPC, for the microgel systems under similar conditions as for Figures 1 and 2. As expected, and in line with the earlier discussion of the results for the variation of the hydrodynamic diameter with added CPC concentration, shown in Figure 2, the strongest (highest affinity) absorption occurs for the poly(NIPAM-co-AAC) microgel particles at $\mathrm{pH} \mathrm{8,} \mathrm{where} \mathrm{the} \mathrm{AAc} \mathrm{moieties}$ are in the ionised $\left(\mathrm{COO}^{-}\right)$form. The next strongest absorption is for the same microgel particles at $\mathrm{pH} 3$, and the weakest absorption is for the poly(NIPAM) microgel particles at $\mathrm{pH}$ 8. Indeed, with these latter two systems the absorption is initially low affinity, which is again consistent with the hydrodynamic diameter data shown in Figure 2. This suggests, for these systems, that once the adsorption to the surface sulfate groups is complete, further absorption into the interior of the microgel particles is then not so easy. Indeed, for the poly(NIPAM) microgel particles, only limited absorption appears to occur until the cmc value $(0.6 \mathrm{mM})$ is approached; thereafter, the absorption becomes more pronounced. This lends support to the suggestion, made in section 2, that micellar-like aggregates of CPC molecules are adsorbing into the microgel particles, and that there may be both electrostatic and steric effects leading to expansion of the "cages" within the microgel particles.

The much higher affinity absorption for the (with 6\% AAc and 6\% BA) at $\mathrm{pH} 8$, seen in Figure 3, is to be expected, because of the electrostatic attraction between the $\mathrm{CPC}$ molecules and the $\mathrm{COO}^{-}$groups within the microgel particles. In Figure 4 the absorption of CPC into these microgel particles is shown as a function of $\mathrm{pH}$, at an initial added CPC concentration value of $0.6 \mathrm{mM}$ (the $\mathrm{cmc}$ value), in this case in a background of $10^{-3} \mathrm{M} \mathrm{KCl}$ solution. As expected, there is a large increase in the absorption of CPC around the $\mathrm{pK}_{\mathrm{a}}$ value for the $-\mathrm{COOH}$ groups ( $\left.\sim \mathrm{pH} 4.8\right)$. 


\section{Release of CPC from Microgel Particles}

From the results presented in Figure 4 for the poly(NIPAM-co-AAC) microgel particles, it would be expected that a switch in $\mathrm{pH}$ from 8 to 3 ought to result in release of

$\sim 70 \%$ of the absorbed CPC back into bulk solution. This situation is broadly verified in Figure 5, where it is shown that $\sim 80 \%$ of the initially absorbed CPC molecules are actually released, at $20{ }^{\circ} \mathrm{C}$. Within the experimental error, estimated at $10 \%$, the data in Figures 4 and 5 are in good agreement. As may be seen from Figure 5, it was found that, upon increasing the temperature to $40{ }^{\circ} \mathrm{C}$, at $\mathrm{pH} 3$, a further $10-15 \%$ of the adsorbed CPC molecules are released. This is presumably due to the contraction of the microgels, caused by the temperature increase, effectively "squeezing" the surfactant molecules out.

The microgel particles were subjected to further CPC absorption / desorption cycles, and both processes were found to be fully reversible, in that the hydrodynamic diameters of the microgel particles always returned to their previous values.

\section{Interaction of CPC with microgel particle monolayers}

Figure 6 compares the apparent thickness of a monolayer of adsorbed poly(NIPAM) microgel particles, as determined from ellipsometry data, to the (DLS) hydrodynamic diameter of the corresponding free microgel particles in a stable dispersion, both as a function of added CPC concentration. Both size parameters show an increase with increasing CPC concentration, but the ellipsometric values are consistently higher than the DLS values. A similar finding was reported previously by us [16] for the same poly(NIPAM) microgel particles, but, in that case, as a function of temperature. It should be remembered that the two experimental techniques require certain simplifying assumptions about the microgel structure, which is known to be inhomogeneous [26, 29], so one must treat any comparisons of size values with caution. Nevertheless, the relative concentration-dependent sizes observed in Figure 6 might be expected, intuitively, since the microgel particles in the deposited monolayer will be somewhat compressed (and, hence, expanded somewhat normal to the substrate surface) during the reswelling (i.e. the cooling) stage of the monolayer deposition process (see the experimental section). 
Figure 7 shows similar comparisons of the hydrodynamic diameter in aqueous dispersion and the ellipsometric thickness of a deposited monolayer for the poly(NIPAMco-AAc) microgel particles on the cationically-modified silica surface, both as a function of CPC initial concentration, at $\mathrm{pH} 3$ (Figure 7a) and at $\mathrm{pH} 8$ (Figure 7b). The ellipsometric data mirror the DLS data in both cases. However, as may be seen in Figure $7 \mathrm{~b}(\mathrm{pH} 8)$ it was possible to determine thickness values for the monolayer over the CPC concentration range 0.4 to $0.8 \mathrm{mM}$, whereas the hydrodynamic diameter values could not be determined over this CPC concentration range, because, as already explained, in dispersion the particles were aggregated. It is clear that the minimum value of the thickness of the microgel monolayer occurs at around 0.5 to $0.6 \mathrm{mM} \mathrm{CPC}$, i.e. very close to the cmc value for CPC in solution.

One difference between the results for the poly(NIPAM) microgel particles and the poly(NIPAM-co-AAc) microgel particles is that, whereas in the former case (Figure 6) the ellipsometric thickness values are consistently higher than the hydrodynamic diameter values, the reverse is true in the latter case (Figure 7). A similar difference was found previously by us [16] for the two types of microgels, but in that work the measurements were made as a function of temperature, rather than CPC concentration. The reason postulated previously [16] for this difference in the observed trends was that, in the case of the poly(NIPAM-co-AAc) microgel particles, there is an attraction between the $\mathrm{COO}^{-}$and $\mathrm{COOH}$ groups, contained within these particles, and the positively charged silica surface, i.e. the microgel particles are partially collapsed onto the surface. This effect is absent for the poly(NIPAM) microgel particles.

Figure 8 shows the apparent thickness values for a monolayer of poly(NIPAM-coAAc) microgel particles, as a function of $\mathrm{pH}$, both in the absence and presence of CPC $(0.6 \mathrm{mM})$, in this case in a background of $10^{-3} \mathrm{M} \mathrm{KCl}$ solution. The most interesting feature, in both cases, is that the thickness passes through a minimum value around $\mathrm{pH} 5$ to 6, just as observed previously when no CPC was present [16]. The previous explanation was that there are two competing effects which determine the thickness of the microgel monolayer on the cationic surface, as the particles become increasingly more negatively charged with increasing $\mathrm{pH}$ : (a) the tendency for them to collapse down onto the surface increases; and (b) they intrinsically want to expand more. Effect (a) 
dominates at lower $\mathrm{pH}$ values and effect (b) at higher $\mathrm{pH}$ values, particularly at $\mathrm{pH}$ values much larger than the $\mathrm{pK}_{\mathrm{a}}$ value of the $\mathrm{COOH}$ moeities.

The observation that, over the whole $\mathrm{pH}$ range studied, the thickness values in the presence of CPC $(0.6 \mathrm{mM})$ are larger than in its absence, most probably reflects the fact that, in the absence of $\mathrm{CPC}$, the microgel particles are net anionic, and are therefore attracted to the cationic surface (and hence are somewhat collapsed), whereas, in the presence of $0.6 \mathrm{mM} \mathrm{CPC}$, the microgel particles would appear, from Figure 1, to be net cationic and therefore tend to expand away from the positively charged surface.

\section{Conclusions}

It has been shown that the addition of CPC to dispersions of poly(NIPAM) and of poly(NIPAM-co-AAc) microgel particles leads to absorption of the CPC into the particles, and corresponding changes in the hydrodynamic diameter of the particles. With the latter set of particles there are strong $\mathrm{pH}$ dependencies. The dependences of the hydrodynamic diameter and the electrophoretic mobility of the microgel particles on the added CPC concentration are strongly correlated with direct CPC absorption measurements. Whether swelling or deswelling occurs depends primarily on electrostatic effects, i.e. on how the net charge developed within the microgel particles changes as a result of the CPC absorption process. A reduced net charge leads to deswelling, whereas an increased net charge leads to swelling. It is also reasoned that swelling may result additionally from "steric" expansion of the gel network by the build-up, above the cmc of CPC (0.6 mM), of micellar type aggregates within the "cages" formed by the cross-linked oligomeric chains of which the particles are comprised. Any contribution from this effect is likely to be secondary, however, for ionic surfactants. Various mechanisms for CPC absorption into the microgel particles are postulated, including electrostatic, polar and hydrophobic interactions.

A comparison has also been made between the effect of added CPC on the hydrodynamic diameter of the free microgel particles in dispersion, and the apparent thickness, as determined from ellipsometry measurements, of deposited monolayers of the same microgel particles on cationically-modified, oxidised silicon surfaces. The 
trends observed with the monolayers are broadly the same as those observed in the bulk dispersion. This leads to the possibility of microgel monolayers being developed for controlled uptake and release applications. Differences in the various trends observed in the two types of system have been successfully rationalised.

\section{Acknowledgements}

We should like to thank SCIC Ltd (Thailand) for funding to enable VN to carry out her PhD studies in the UK. We also thank the Ministry of Higher Education of Saudi Arabia for providing a $\mathrm{PhD}$ scholarship for IB.

\section{References}

[1] Murray, M.J.; Snowden, M.J., Adv. Colloid Interface Sci., 1995,54, 73.

[2] Saunders, B.R; Vincent, B, Adv. Colloid Interface Sci., 1999, 80, 1.

[3] Saunders, B.R.; Vincent, B., Encyclopedia of Surface and Colloid Science, Marcel Dekker, USA, 2002, 4544.

[4] Morris, G.E.; Vincent, B; Snowden, M.J., J.Colloid Interface Sci., 1997, 190, 198.

[5] Morris, G.E.; Vincent, B; Snowden, M.J., Prog. Colloid Polymer Sci., 1997, 105, 16.

[6] Bradley, M.; Vincent, B., Langmuir, 2005, 21, 8630.

[7] Bradley, M.; Vincent, B; Warren, N; Eastoe, J; Vesperinas, A., Langmuir, 2006, $22,101$.

[8] Bradley, M.; Ramos, J.;Vincent, B., Langmuir, 2005, 21, 1209.

[9] Routh, A; Fernandez-Nieves, A; Bradley, M; Vincent, B, J. Phys. Chem. B, 2006, $110,12721$.

[10] Bradley, M.; Bruno, N.;Vincent, B., Langmuir, 2005, 21, 2750.

[11] Tam,K.C.; Ragaram, S.; Pelton, R.H., Langmuir, 1994,10, 418

[12] Wang,G.; Pelton, R.H.;Zhang, R; Colloids Surfaces A, 1999, 153, 335.

[13] Woodward, N.C.; Chowdhry, B.Z.; Leharne, S.A. ; Snowden, M.J., Euro. Polymer J., 2000, 36, 1355.

[14] Nafee, N.A.; Boraie, M.A.; Ismail, F.A.; Mortada, L.M., Acta Pharma, 2003,53, 199

[15] Densmore, C.G.; Robinson, T.W.; Smith, B.F.; Lewis, R.E., J.App. Polymer. Sci., 2006, 99, 3153

[16] Nerapusri, V.; Keddie, J.L.; Vincent, B; Bushnak, I.A, Langmuir, 2006, 22, 5036.

[17] Pelton, R.H.; Chibanate P, Colloids Surfaces, 1986, 20, 247.

[18] Keddie, J.L., Curr. Opin. Coll. Interf. Sci., 2001, 6, 102.

[19] Shubin, V.; Linse P., J. Phys.Chem., 1995, 99, 1285.

[20] Amiel, C.; Sikka, M.; Schneider, J.W., Macromolecules, 1995, 28, 3125.

[21] Minko, S.; Sidorenko, A.; Stamm, M., Macromolecules, 1999, 32, 4532.

[22] Styrkas, D.A. et al., Langmuir, 2000, 16, 5980. 
[23] Smith, T., J. Optical Soc. Amer., 1968, 58, 69.

[24] Styrkas, D.A., Polymer Surfaces and Interfaces III, ed. Richards, R.W.; Peace, S.K., John Wiley, Chichester, 1999, 1.

[25] Marquardt, D.W., J.Soc.Ind. Appl. Math., 1963, 11, 431.

[26] Mason, T.G.;Lin, M.Y., Phys. Rev. E., 2005, 71, 40801

[27] Skerjanc, J.; Kogej, K; Vesnaver, G., J. Phys. Chem., 1988, 92, 6382.

[28] Mears, S.J.; Deng, Y.; Cosgrove, T.; Pelton, R.H., Langmuir, 1997, 13, 1901.

[29] Fernandez-Nieves, A.; de las Nieves, F.J.; Fernandez-Barbero, A., J. Chem. Phys, 2004 120, 374. 


\section{Figure Captions}

Figure 1. Electrophoretic mobility as function of added CPC concentration for the dispersed microgel particles. ( $)$ poly(NIPAM) (with 1\%BA) at pH8; (•) poly(NIPAM-co-AAc) (with 6\%AAc and 6\%BA) at $\mathrm{pH} 3 ;(\mathrm{O})$ poly(NIPAM-co-AAc) (with 6\%AAc and 6\%BA) at $\mathrm{pH} 8$.

Figure 2. Hydrodynamic diameter as function of added CPC concentration for the dispersed microgel particles. ( $)$ poly(NIPAM) (with 1\%BA) at pH 8; ( $\bullet$ poly(NIPAMco-AAc) (with 6\%AAc and 6\%BA) at $\mathrm{pH} 3$; (O) poly(NIPAM-co-AAc) (with 6\%AAc and $6 \% \mathrm{BA})$ at $\mathrm{pH} 8$.

Figure 3. Absorbed amount of CPC, as function of equilibrium CPC concentration, for microgel particles. ( ) poly(NIPAM) (with 1\%BA) at pH8; (•) poly(NIPAM-co-AAc) (with 6\%AAc and 6\%BA) at $\mathrm{pH} 3$; (O) poly(NIPAM-co-AAc ) (with 6\%AAc and $6 \% \mathrm{BA})$ at $\mathrm{pH} 8$.

Figure 4. Absorbed amount of $\mathrm{CPC}$ as function of $\mathrm{pH}$ for poly(NIPAM-co-AAc) microgel particles (with 6\%AAc and 6\%BA) at an CPC concentration of $0.6 \mathrm{mM}$ and in $10^{-3} \mathrm{M} \mathrm{KCl}$ solution.

Figure 5. Absorbed and released amount of $\mathrm{CPC}$, as function of $\mathrm{CPC}$ equilibrium concentration, for poly(NIPAM-co-AAc) microgel particles (with 6\%AAc and 6\%BA). (ם) absorbed amount at $\mathrm{pH} 8$ and $20{ }^{\circ} \mathrm{C}$; (•) released amount at $\mathrm{pH} 3$ and $20{ }^{\circ} \mathrm{C}$; (O) further released amount at $\mathrm{pH} 3$ upon increasing the temperature to $40{ }^{\circ} \mathrm{C}$.

Figure 6. Hydrodynamic diameter of absorbed poly(NIPAM) microgel particles (1 wt $\%$ BA) in aqueous dispersion and the ellipsometric thickness of a monolayer of these particles at a cationically-modified silica interface, both as a function of initial CPC concentration, at $\mathrm{pH} 8$ and $20{ }^{\circ} \mathrm{C}$.

Figure 7. Hydrodynamic diameter of absorbed poly(NIPAM-co-AAc) microgel particles (6 wt\% AAc and $6 \mathrm{wt} \% \mathrm{BA}$ ) in aqueous dispersion and the ellipsometric thickness of a monolayer of these particles at a cationically-modified silica interface, both as a function of initial CPC concentration, at $20{ }^{\circ} \mathrm{C}:$ (a) at $\mathrm{pH} 3$, (b) at $\mathrm{pH} 8$.

Figure 8. Ellipsometric thickness of a monolayer of poly(NIPAM-co-AAc) microgel particles (with 6\% AAc and 6\%BA), as a function of $\mathrm{pH}\left(20{ }^{\circ} \mathrm{C}\right.$, and in $10^{-3} \mathrm{M} \mathrm{KCl}$ solution). ( $\bullet$ at an initial CPC concentration of $0.6 \mathrm{mM} ; \diamond)$ without CPC. 


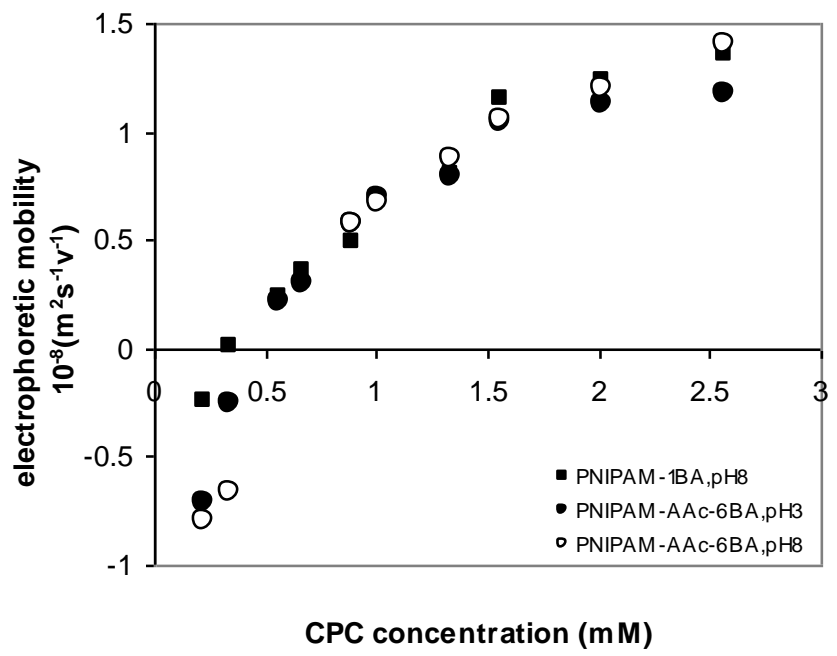

Figure 1.

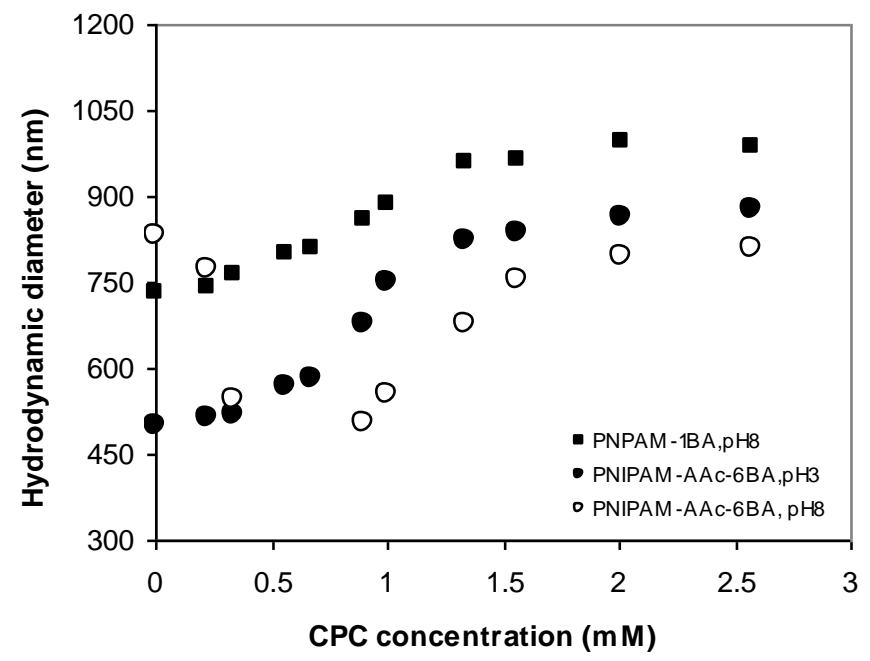

Figure 2. 
Published in Langmuir (2007) 23(19): 9572-9577

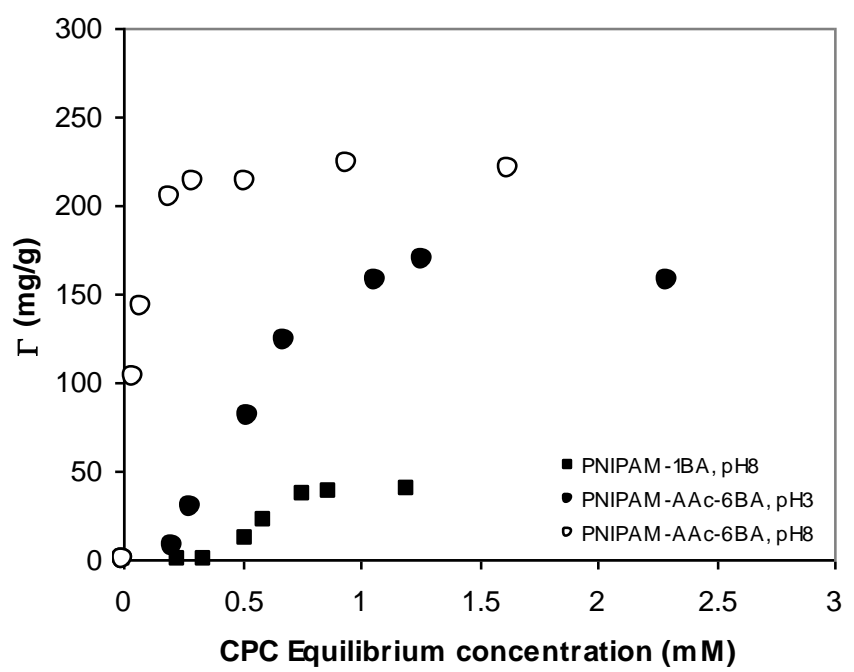

Figure 3.

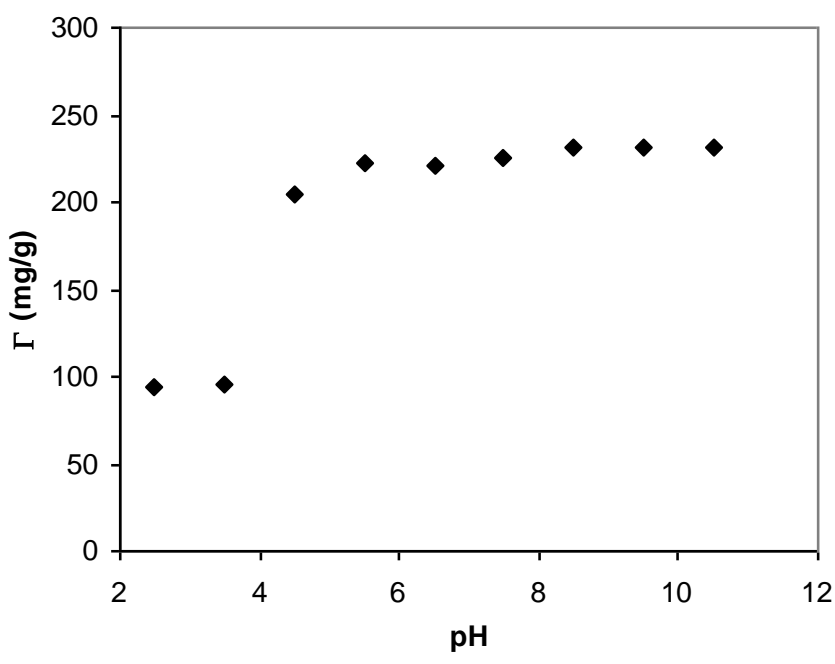

Figure 4. 
Published in Langmuir (2007) 23(19): 9572-9577

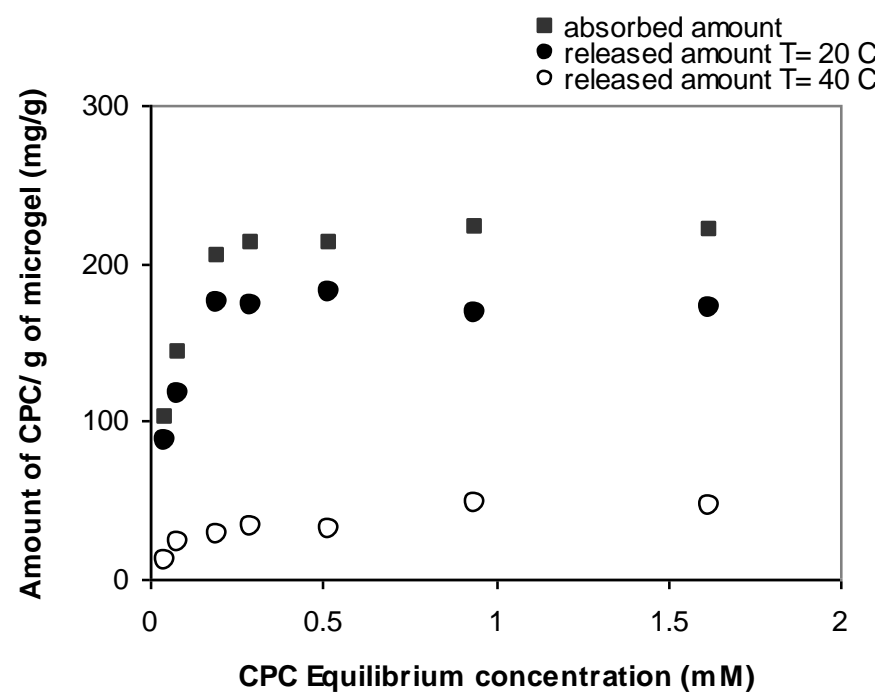

Figure 5.

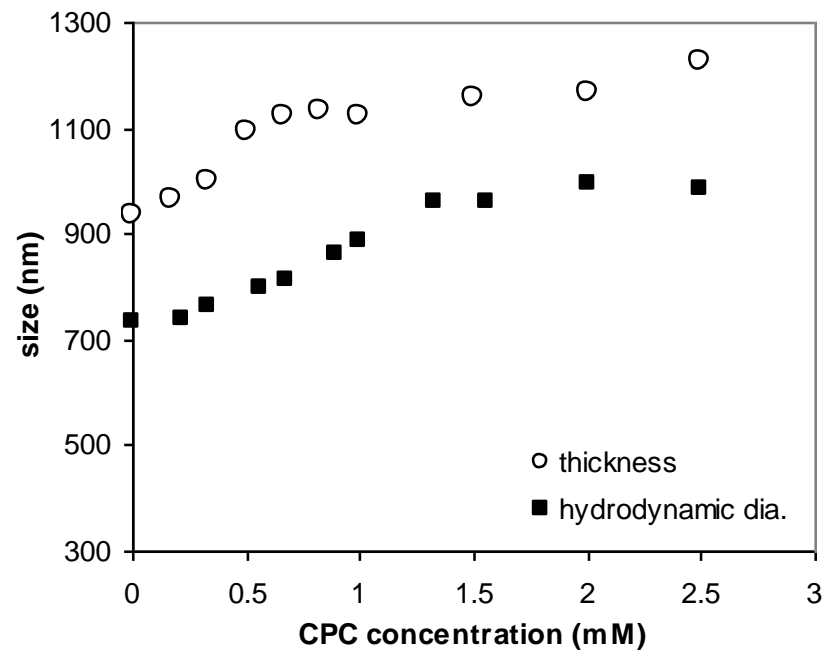

Figure 6. 
Published in Langmuir (2007) 23(19): 9572-9577
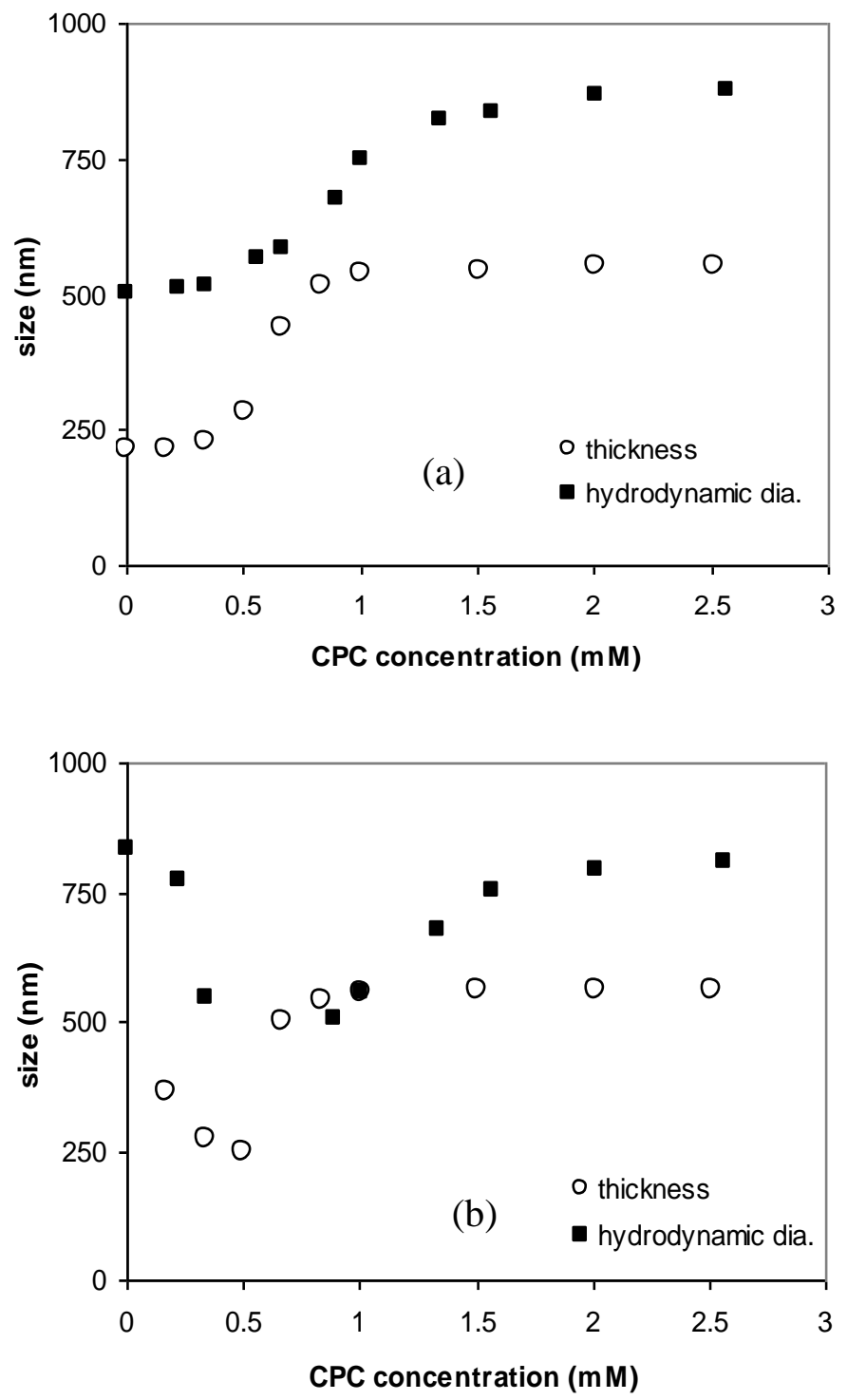

Figure 7. 
Published in Langmuir (2007) 23(19): 9572-9577

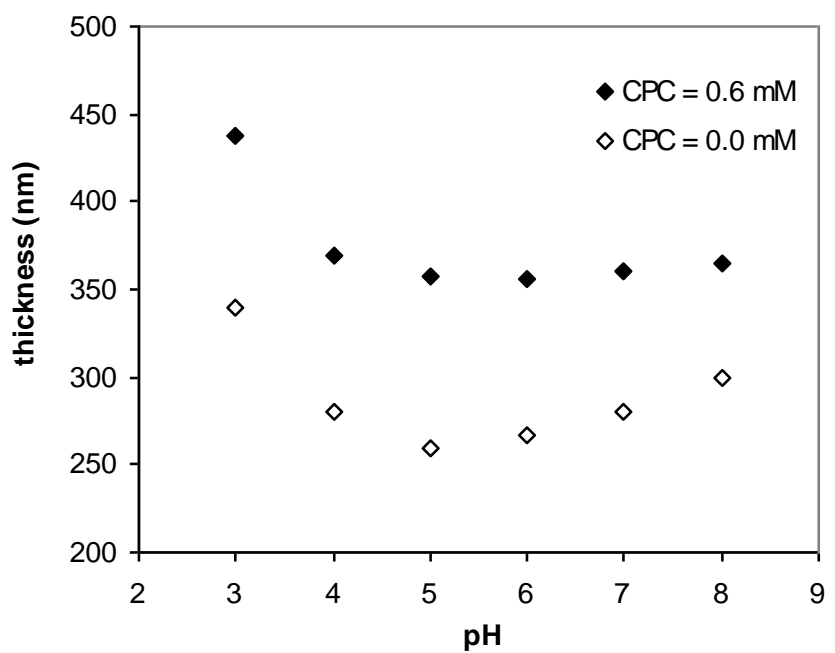

Figure 8. 